\title{
LISTA ANOTADA DE LAS ESPECIES DE EUPHORBIACEAE PARA EL QUINDÍO
}

\section{ANNOTATED LIST OF EUPHORBIACEAE SPECIES FOR QUINDÍO}

\author{
Carlos Alonso Maya Lastra ${ }^{1}$, Carlos Alberto Agudelo Henao ${ }^{2}$
}

${ }^{1}$ Estudiante Maestría en Biología Vegetal de la Universidad del Quindío

${ }^{2}$ Profesor Programa de Biología, Universidad del Quindío. Centro de Estudios e Investigaciones en Biodiversidad y Biotecnología CIBUQ.

Fecha de recibido: Febrero 3 de 2010

Fecha de aceptado: Junio 9 de 2010

Correspondencia: Centro de Estudios e Investigaciones en Biodiversidad y Biotecnología - CIBUQ, Universidad del Quindío, Carrera 15 calle $12 \mathrm{~N}$, Armenia (Quindío). Correo electrónico: camayal@gmail.com

\section{RESUMEN}

Se realizó el estudio taxonómico de la familia Euphorbiaceae en el departamento del Quindío, Colombia, el cual fue desarrollado con el fin de conocer las especies presentes en la región, para lo cual se revisaron y determinaron 423 ejemplares de la colección del Herbario Universidad del Quindío (HUQ). Para esta región se reconocieron 16 géneros, de los cuales Euphorbia y Acalypha reportaron el mayor número de especies, 9 y 7 respectivamente, seguidos por Croton (5 spp.), Dalechampia (3 spp.), Alchornea (2 spp.), Mabea (2 spp.), Jatropha (2 spp.) y Chamaesyce (2 spp.) con un número menor de especies y 8 géneros monoespecíficos, Ricinus, Dysopsis, Tetrorchidium, Manihot, Codiaeum, Caperonia, Plukenetia y Sapium. A nivel de especies se encontraron 40 en total, de las cuales 14 fueron nuevos registros para el departamento, destacnadose entre estas Dalechampia canescens, $D$. cissifolia, Plukenetia volubilis y Croton xalapensis, siendo esta última un nuevo registro para Colombia. Se confirmó además la presencia de Acalypha platyphylla y de A. setosa en la zona y se identificó a A. diversifolia, A. macrostachya y Euphorbia heterophylla como las especie con mayor distribución en el departamento, encontrándonse en los 12 municipios, y a Dalechampia canescens, Dysopsis paucidentata y Euphorbia peplus como las de menor distribución, ya que sólo se localizan en uno o dos municipios.

Palabras claves. Euphorbiaceae, flora, taxonomía, Quindío, Colombia, lista anotada,

\section{ABSTRACT}

We present an annotated list for all species of Euphorbiaceae that occurs in department of Quindio. We revised and determined 423 specimens from the Herbarium collection of University of Quindio (HUQ). For Quindio we found that Euphorbiaceae is represented by 16 genera: Euphorbia and Acalypha as the major genus with 9 and 7 species respectively, followed by Croton (5 spp), Dalechampia (3 spp), Alchornea (2 spp), Mabea (2 spp), Jatropha (2 spp) and Chamaesyce (2 spp), and 8 monospecific genus, Ricinus, Dysopsis, Tetrorchidium, Manihot, Codiaeum, Caperonia, Plukenetia and Sapium. In was found 40 species in total, of which 14 were new reports for Quindío: Dalechampia canescens, D. cissifolia, Plukenetia volubilis and Croton xalapensis, the latter is a new report for Colombia. We confirmed the presence of Acalypha platyphylla and A. setosa and we also identified A. diversifolia, A. macrostachya and Euphorbia heterophylla as species with wide distribution based on findings in 12 localities of Quindío; in contrast, Dalechampia canescens, Dysopsis paucidentata and Euphorbia peplus only were found in one or two localities.

Keywords: Euphorbiaceae, flora, taxonomy, Quindío, Colombia, South America, annotated list.

\section{INTRODUCCIÓN}

Euphorbiaceae es una familia compuesta por un número cercano a 8100 especies (Webster et al. 1999). Al tratarse de una familia con un elevado número de taxones, su organización se hace más compleja surgiendo problemas taxonómicos como la delimitación familiar, la composición subfamiliar y la organización infragenérica. Sus constantes cambios jerárquicos a lo largo de la historia, han dejado una impresión de desorganización, con la cual generalmente es reconocida la familia; sin embargo, gracias a algunos de los últimos trabajos, la familia posee una estructura taxonómica estable en comparación con otros grupos naturales.

Los anteriores aspectos fueron los que nos motivaron a realizar un estudio taxonómico del grupo para aportar al conocimiento de la biodiversidad específica de Euphorbiaceae en el departamento del Quindío y mediante una lista anotada extender el saber acerca de esta familia, así como también responder las siguientes preguntas ¿Cuáles y cuántas especies se encuentran en el Quindío? y ¿Cómo se distribuyen?, además de brindar una clave dicotómica para la identificación de los géneros presentes en el departamento. Esta lista anotada de especies se construyó basándose en la revisión taxonómica y morfológica de la familia para el Quindío (Maya \& Agudelo 2007), trabajo de grado Laureado del Programa de Licenciatura en Biología de la Universidad del Quindío. 


\section{MATERIALES Y MÉTODOS}

Área de estudio y fase de campo. Comprendió todo el departamento del Quindío (12 municipios), el cual está localizado en la región centro-occidental del país, entre los

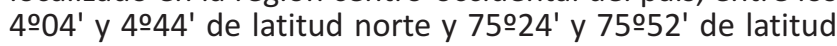
oeste. Para la fase de campo se realizó un muestreo aleatorio entre febrero de 2006 y 2007, en tal periodo se efectuaron diez salidas de 1 a 4 días duración a los diversos tipos de hábitats existentes en los municipios del Departamento, correpondiente a una forma de inventario sin unidad de muestréo específica (Villarreal et al. 2004). De cada espécimen se tomaron dos muestras completas que incluían partes vegetativas y reproductivas. Cada muestra de un tamaño aproximado de $40 \mathrm{~cm}$. En total se colectaron 56 ejemplares y los cuales se evaluaron junto con otros 367 que estaban previamente almacenados en HUQ.

Fase de Herbario. Las muestras colectadas en campo fueron procesadas de acuerdo al método planteado por Lot \& Chiang (1987), el cual consiste en un el prensado en dos láminas de aluminio plegadas, el secado en un horno a una temperatura estable de 70 으 y el montaje en cartulina dúplex de $30 \times 40 \mathrm{~cm}$. Todos los ejemplares fueron identificados con una etiqueta estándar (incluye datos morfológicos, colores de algunos órganos, localización, altitud, fecha de colección, colector y su número) para llevarlas al HUQ. Cada uno de los 423 espécimenes estudiados, fueron determinados con ayuda de claves (Rentería 1994, Cuatrecasas 1957, Standley \& Steyermark 1949, entre otros) de acuerdo a las características morfológicas identificadas en ellos, además fueron confrontados sus rasgos morfológicos con las descripciones existentes en la literatura (Webster \& Burch 1968, Cardiel 1995, Macbride 1951, entre otros).

La lista anotada de especies se construyó con datos tales como: el nombre de los géneros con su referencia bibliográfica, la especie tipo, etimología, composición, distribución y comentarios generales, posteriormente se presenta cada especie en orden alfabético con datos como hábito, distribución, comentarios generales y un espécimen representativo. También se incluyó una clave dicotómica para los géneros de Euphorbiaceae encontrados en el Quindío.

\section{CLAVE PARA LOS GÉNEROS DE EUPHORBIACEAE PRESENTES EN EL QUINDÍO}

\section{Lianas}

2. Fruto tricoco, globoso, $0.5-1 \mathrm{~cm}$ de alto, menores a $2 \mathrm{~cm}$ de diámetro.

2. Fruto tetracoco, alado, $4.5-5 \mathrm{~cm}$ de alto, $5-6 \mathrm{~cm}$ de diámetro.

1. Árboles, arbustos o hierbas.

3. Anteras tetratecadas.

3. Anteras bitecadas a monotecadas.

4. Inflorescencias en ciatio.

5. Hojas alternas, verticiladas u opuestas (cuando opuestas base simétrica).

5. Hojas opuestas (siempre con base asimétrica).

4. Inflorescencias cimosas, espiciformes, racemosas o flores solitarias.

6. Glándulas foliares (o peciolares) ausentes.

7. Hojas palmatilobuladas, irregulares o variables en forma.

8. Perianto masculino representado por 3 brácteas.

8. Perianto masculino representado por tépalos o sépalos.

9. Sépalos y pétalos indiferenciables (tépalos); frutos globosos con aristas alada.

9. Sépalos y pétalos diferenciables; frutos trilobulados, sin aristas aladas.

7. Hojas enteras no lobuladas, regulares, estables.

10. Hierbas postradas; hojas con 5-7 crenaciones; flores solitarias.

10. Hierbas no postradas y arbustos; hojas con 13-57 pares de dientes; flores en inflorescencias.
Codiaeum

Manihot

Dalechampia

Plukenetia

Tetrorchidium

Chamaesyce

Jatropha

Dysopsis

Acalypha
Euphorbia 
6. Glándulas foliares (o peciolares) presentes

11. Setas glandulosas sobre toda la planta.

Caperonia

11. Setas ausentes.

12. Haz las hojas y pecíolos pubescentes.

13. Tricomas estrellados, rotados a escamosos, ovario tricarpelar, estilos menores a $0.8 \mathrm{~cm}$ de largo

Croton

13. Tricomas simples, ovario bicarpelar, estilos mayores a $1.5 \mathrm{~cm}$ de largo.

Alchornea

12. Haz de las hojas y pecíolos glabros.

14. Arbustos sin látex, con capa velutina; hojas palmatilobuladas; pecíolo peltado.

Ricinus

14. Árboles con látex, sin capa velutina; hojas enteras, oblongas a elípticas; pecíolo basifijo.

15. Fruto piriforme, apicalemente mucronado, exocarpo glabro, liso y lustroso.

Sapium

15. Fruto globoso, apicalmente enlongado a achatado, exocarpo pubescente a tomentoso, tricomas ocre, ca. $0.1 \mathrm{~mm}$ de largo.

Mabea

\section{LISTA ANOTADA}

A continuación se presenta la lista anotada de las 40 especies de Euphorbiaceae encontradas en el Quindío haciendo anotación puntual de su forma biológica, distribución, rango altitudinal y están enumeradas de forma consecutiva.

Acalypha L., Sp. Pl. 1003. 1753.

Especie tipo. Acalypha virginica L. - Sp. PI. 2: 1003.

Etimología. Linneo derivó el nombre genérico del griego akalos que significa desagradable, y aphe: tacto (Cardiel 1995).

Es el cuarto género más grande de la familia Euphorbiaceae con aproximadamente 450 especies, distribuidas en la zona tropical y subtropical, encontrándose además un pequeño grupo de especies en zonas templadas. (Webster 1994). En Colombia Cardiel (1995) registró 24 especies las cuales ocupan zonas andinas y costeras, mostrando menor riqueza en zonas amazónicas y desérticas. En este estudio se reportan 7 especies $A$. amentacea, A. arvensis, A. diversifolia, A. hispida, A. macrostachya, A. platyphylla y A. setosa.

1. Acalypha amentacea Roxb, Smithsonian Contr. Bot. 45 : 10. 1980

Arbusto monoico, 1-1.5 m de altura. Por ser una planta ornamental, se encuentra limitada a estar en jardines y parques, razón por la cual su distribución es reducida, hallándose solamente en la ciudad de Armenia entre los 1375-1560 m de altitud.

Espécimen representativo. Armenia: Parque Los Aborígenes, 1560 m, 17 abr 1993, Perea 7 (HUQ).

2. Acalypha arvensis Poepp. in Poepp. \& Endl., Nov. Gen. Sp.
Pl. 3: 21. 1841. Figura 1.

Hierba monoica de hasta $0.4 \mathrm{~m}$ de altura. Suele encontrase en bordes de carreteras o asociadas a cultivos como el de café. Hasta ahora se ha localizado en los municipios de Salento, Armenia y Montenegro, a una altitud que oscila entre los 1150-1860 m.

Espécimen representativo. Armenia: Vda Murillo, Fca Villa Adriana, cerca de cultivo de Musa sp, 1020 m, 30 oct 2006, Maya 38(HUQ).

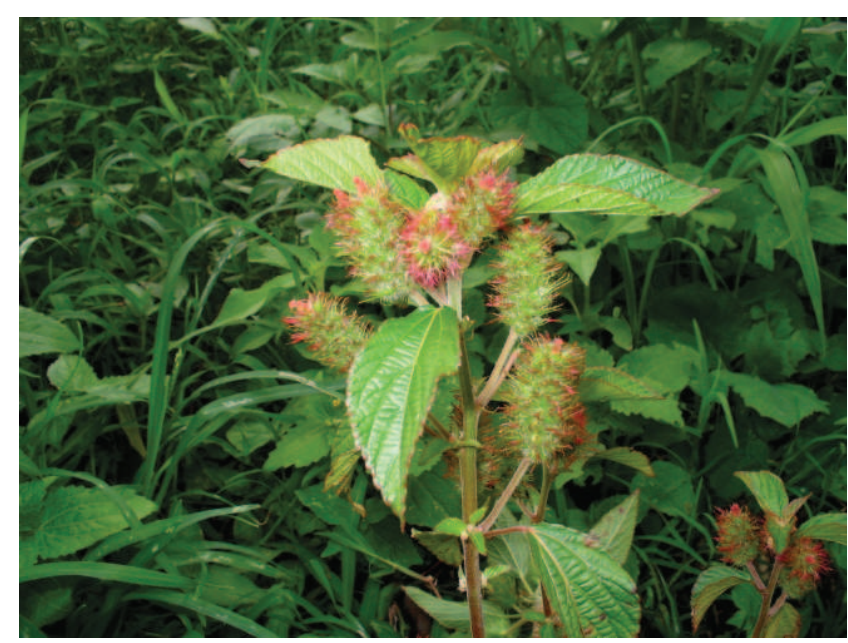

Figura 1. Hábito de Acalypha arvensis

3. Acalypha diversifolia Jacq., Pl. Hort. Schoenbr. 2: 63, t. 244. 1797.

Arbusto con ramificación temprana, presenta ramas péndulas, monoica, 1-6 $\mathrm{m}$ de altura. Se ha encontrado en los municipios de Armenia, Calarcá, Circasia, Córdoba, Filandia,

Rev. Invest. Univ. Quindío (20): 126 - 136. Armenia - Colombia 
Pijao, Quimbaya y Salento. Las altitudes a las que crece oscilan entre los 1150 - $2410 \mathrm{~m}$, ubicándose en todo los pisos altitudinales que comprende el Quindío, excepto el paramuno.

Espécimen representativo. Circasia: Vda Barcelona Alta, Fca Buenos Aires, 1690 m, 23 mar 1991, Agudelo et al. 1068 (HUQ).

4. Acalypha hispida Burm. f. Fl. Indica nec non Prodromus Florae Capensis 303, pl. 61, f. 1.1768.

Arbusto de hasta $2 \mathrm{~m}$ de alto, ramificado basalmente. La presencia de dicha especie, se encuentra ligada a la frecuencia de siembra de la misma en los jardines por ser de uso ornamental, pudiéndose hallar en los municipios de Calarcá, Circasia, La Tebaida, Montenegro y Quimbaya.

Espécimen representativo. Armenia: Parque Uribe, 1375 m, 13 mar 1993, Cardona 2 (HUQ).

5. Acalypha macrostachya Jacq. Pl. Hort. Schoenbr. 2: 63, pl. 245. 1797.

Arbusto monoico, 2,5-6 m de altura, $10-17 \mathrm{~cm}$ de CAP. Se encuentra distribuida en nueve de los doce municipios del departamento, mostrando una gran adaptación tanto a ambientes cálidos como templados (Armenia, Calarcá, Circasia, Filandia, La Tebaida, Montenegro, Pijao, Quimbaya y Salento). Igualmente su posición altitudinal es variada, creciendo desde los $1090-2170 \mathrm{~m}$. Estas plantas suelen estar ubicadas en bordes de carreteras y caminos principalmente, aunque en ocasiones se les suele encontrar cerca de guaduales y a pequeños cuerpos de agua.

Espécimen representativo. Filandia: Sitio El Salado, rivera del río Barbas, 1400 m, 12 jul 1985, Arbelaez et al. 951 (HUQ).

\section{Acalypha platyphylla Müll. Arg. Linnaea 34: 6. 1865.}

Hierbas o pequeños arbustos, monoicas, 1-4 m de altura, látex ausente. Esta es una de las especies del género Acalypha con mayor restricción que se ha encontrado en el departamento, ella se ubica en algunas zonas de los municipios de Calarcá, Circasia y Salento; igualmente su distribución altitudinal es restringida entre los 1600-2200 m.

Espécimen representativo. Calarcá: Corregimiento Qda Negra, Vda Vista Hermosa, Fca La Floresta, 1650-2150 m, 2 mar 1991, Agudelo et al. 873 (HUQ).

7. Acalypha setosa A. Rich. in Sagra, His. Fis. Cuba, Bot. 11: 204. 1850.

Hierba monoica, 20 a $50 \mathrm{~cm}$ de altura. Solo se ha encontrado en el municipio de La Tebaida, crece en bordes de camino y en lotes abandonados. Su rango altitudinal es restringido (1170 m hasta $1340 \mathrm{~m})$.

Espécimen representativo. La Tebaida: Vda Murillo, Fca La Sonia, borde de carretera y cafetales, 1175 m, 2 oct 1996, Agudelo et al. 3381 (HUQ).
Alchornea Sw., Prodr. 6: 98. 1788.

Especie tipo. Alchornea latifolia Sw. - Nova Genera et Species Plantarum seu Prodromus 98. 1788.

Etimología. Alchornea es dado en homenaje al botánico inglés Stanesby Alchorne (Pax \& Hoffmann, 1914).

Webster (1994) estima un número cercano a las 50 especies para todo el mundo. Secco (1999) reporta para el neotrópico 22 especies, anotando la alta diversidad de Alchornea para Colombia, en donde se estima un total de 16 especies al igual que Rentería (1994). Por su parte Murillo (2004) hace el último estimativo para Colombia de 19 especies. Para el departamento del Quindío, se contaba con un reporte realizado por Vargas (2002) en el cual se registraban cuatro especies, $A$. grandiflora, $A$. grandis, $A$. glandulosa y $A$. coelophylla, estas fueron reevaluadas en este trabajo, reduciéndolas a $A$. glandulosa, ya que la estructura floral ilustrada en las claves del manuscrito, no reflejan diferencias significativas para considerar estos cuatro taxones. Adicional a este, se encontró A. latifolia.

8. Alchornea glandulosa Poepp. Nov. Gen. Sp. PI. 3: 18, pl. 221. 1841.

Árbol, 2-15 $\mathrm{m}$ de alto, $12-82 \mathrm{~cm}$ de CAP, ramificado suprabasalmente. Esta espécie se reportó en Armenia, Calarcá, Circasia, Filandia, Pijao y Salento, entre 1500-2200 $\mathrm{m}$, los localizados en altitudes menores a los $1600 \mathrm{~m}$, son plantados.

Espécimen representativo. Circasia: Vda Buenavista Alto, río el Roble, 1530 m, 5 jul 1985, Arbeláez et al. 929 (HUQ).

\section{Alchornea latifolia Sw., Prodr. 98. 1788.}

Árbol monoico, 4-20 m de alto, $72-220 \mathrm{~cm}$ de CAP, algunas veces ramificado basalmente. $A$. latifolia se encuentra distribuida, a lo largo de la zona templada del departamento del Quindío, incluyendo los municipios de Armenia, Buenavista, Calarcá, Circasia, Córdoba, Filandia, Génova, Pijao y Salento, entre 1500-2000 m; se suele hallar en lugares sombríos, dentro de los relictos boscosos.

Espécimen representativo. Córdoba: Vda El Crucero, en cafetal, s.a., 15 ago 2000, Marulanda 26 (HUQ).

Caperonia A. St.-Hil. Hist. PI. Remarq. Bresil. 244. 1826

Especie tipo. Caperonia castaneifolia (L.) A. St.-Hil. Histoire des plantes les plus remarquables du Bresil. 245. 1826.

Etimología. En honor a Noël Caperon, farmacéutico de Orleans, Francia (Smith et al. 1988).

Este género se distribuye principalmente en Sur y Centroamérica, incluyendo también África y la zona occidental de India; se incluyen en él ca. 35 especies (Webster et al. 1999), de las cuales tres aproximadamente se encuentran en Colombia (Murillo 2004). En este trabajo se reporta Caperonia palustris como la única especie del género presente en el departamento del Quindío. 
10. Caperonia palustris (L.) A. St.-Hil. Hist. Pl. Remarq. Bresil. 245. 1826.

Hierba $30-40 \mathrm{~cm}$ de alto, monoica, ramificada monopódicamente. Se encuentra limitada a las áreas cálidas del departamento, hacia el occidente y el suroccidente de éste. Se halló en los municipios de La Tebaida y Montenegro. Altitudinalmente su rango de distribución no es tan amplio, localizándose cerca a los $1000 \mathrm{~m}$.

Espécimen representativo. Montenegro: Fca Los Alpes, monte Veráguas, 1100 m, 23 sep 2001, Vélez et al. 7195 (HUQ).

\section{Chamaesyce Gray, Nat. Arr. Brit. Pl. 2: 260. 1821.}

Especie tipo. Chamaesyce maritima Gray Nat. Arr. Brit. PI. 2: 260. 1821

Etimología. Chamaesyce proviene de dos palabras griegas chamai, en el suelo y skyon, higo. Este era el nombre dado a plantas similares a este género (Contr. Gard. Dave, 2007).

Chamaesyce se distribuye principalmente en el neotrópico y en los subtrópicos, consta de 250 especies a nivel mundial (Webster et al. 1999). Para Colombia, Murillo (2004), estimó 6-8 especies, nombrándolas como Euphorbia conservando la ubicación de Chamaesyce como sección. Para el departamento del Quindío se encontraron 2 especies, Chamesyce hirta y $C$. hyssopifolia; y se considera que se puede encontrar la especie C. prostrata.

11. Chamaesyce hirta (L.) Millsp. Publ. Field Columbian Mus., Bot. Ser. 2(7): 303. 1909.

Hierba rastrera, postrada, monoica. Látex blanco, viscoso, oxida a rojizo. Esta especie, tiene una amplia distribución; en el departamento se le puede encontrar en municipios bajos y cálidos como La Tebaida, Quimbaya, Montenegro, al igual que en municipios altos y templados como Armenia, Buenavista, Calarcá, Filandia y Salento. Se asocia principalmente a zonas pedregosas como bases de barrancos y carreteras no pavimentadas. El rango altitudinal en el cual se encuentra esta especie en el departamento es $900-2860 \mathrm{~m}$

Espécimen representativo. Montenegro: Cerca de la reserva natural La Montaña del Ocaso, antes de Playa Azul, en la carretera, 900 m, 10 may 2006, Maya 13 (HUQ).

12. Chamaesyce hyssopifolia (L.) Small Bull. New York Bot. Gard. 3(11): 429. 1905.

Hierba escandente, ramificada basalmente, $30-60 \mathrm{~cm}$ de altura, Látex abundante, blanco, oxida a rojizo. Esta especie fue encontrada en los municipios de Armenia, La Tebaida y Quimbaya, municipios donde las temperaturas suelen ser de altas a moderadas, constituyen además la parte baja del Quindío y son limítrofes con la cuenca del río La Vieja. Se distribuye de los 1050-1330 m.

Espécimen representativo. La Tebaida: Hda Villa Eloisa, borde de carretera, 1240 m, 16 mar 1991, Agudelo \& Varela 1791 (HUQ).
Codiaeum Rumph. ex A. Juss. Euphorb. Gen., tabulis aeneis 18 illustratum 33. 1824.

Especie tipo. Codiaeum variegatum (L.) A. Juss. - De Euphorbiacearum Generibus Medicisque earumdem viribus tentamen, tabulis aeneis 18 illustratum 80, 111, pl. 9, f. 30. 1824.

Etimología. La palabra Codiaeum la utilizó el naturalista Holandés G. E. Rumphius, para referirse a las plantas llamadas codebos en Malasia, siendo este una latinización de un vocablo asiático (Contr. Gard. Dave, 2007).

Codiaeum es un género Austraasiático de ca. 15 especies, distribuyéndose desde Bordeo y las Filipinas hasta Nueva Guinea, Australia tropical, Nueva Caledonia y las islas del pacífico (Webster 1994). En América se encuentra distribuido únicamente como ornamental, siendo el caso también para Colombia y respectivamente para el Quindío, encontrándose solamente la especie Codiaeum variegatum.

13. Codiaeum variegatum (L.) A. Juss. Euphorb. Gen., tabulis aeneis 18 illustratum 80, 111, pl. 9, f. 30. 1824.

Arbusto monoico, 1-2.7 $\mathrm{m}$ de alto, ramificado monopódicamente. Todos los ejemplares de esta planta fueron colectados en el municipio de Armenia entre 1500 1540 m, en parques y zonas ornamentadas con vegetación.

Espécimen representativo. Armenia: Barrio LoS Guaduales, bloque 6, 1500 m, 24 may 1996, Angulo 2 (HUQ).

Croton L., Sp. PI. 2: 1004-1005. 1753.

Especie tipo. Croton aromaticus L. Species Plantarum 2: 1004. 1753.

Etimología. El nombre Croton, proviene de la palabra griega kroton que significa garrapata, ya que algunas semillas de este género se asemejan a estos animales (Contr. Gard. Dave, 2007).

Croton es un género de distribución cosmopolita, el cual muestra un mayor número de especies en la zona tropical del mundo, compuesto por ca. 1200 especies (Webster et al. 1999). En Colombia existen aproximadamente 80 especies (Murillo 2004), siendo uno de los géneros con mayor número de especies. Mediante este trabajo se logró determinar un total de 5 especies ( $C$. hirtus, $C$. smithianus, $C$. speciosus, $C$. xalapensis, C. sp.), registrándose un nuevo registro para Colombia (C. xalapensis).

\section{Croton hirtus L'Hér. Stirp. Nov. 17, pl. 9. 1785.}

Hierba (16-)30-60(-85) cm de alto, monoica, látex ausente. Puede estar limitado a lugares cálidos, en potreros, bordes de caminos, surcos o canaletas; ha sido hallada en los municipios de Armenia, Buenavista, Calarcá, La Tebaida, Montenegro, Quimbaya y Salento. Las alturas a las que se suele encontrar $C$. hirtus se encuentran entre 950-1840 m, siendo la franja de mayor ocurrencia entre 950-1300 m.

Espécimen representativo. Montenegro: Ribera del río La Vieja, en potrero, zona soleada, 900 m, 10 may 2006, Maya 8(HUQ). 
15. Croton smithianus Croizat, J. Arnold Arbor. 21: 93.1940.

Árbol 8-10 m de alto. Látex pardo claro a amarillento, viscoso. Se ha encontrado el municipio de Filandia, a una altura promedio de $1590 \mathrm{~m}$, suele hallarse en los potreros o cerca de cuerpos pequeños de agua.

Espécimen representativo. Filandia: Carretera FilandiaCircasia, margen izquierda, Qda Los Medios, 1590 m, 16 dic 1986, Arbeláez et al. 1900 (HUQ).

\section{Croton speciosus Müll. Arg. Linnaea 34: 83. 1865.}

Árbol 6-8 m de alto. Látex amarillo a amarillo verdoso, oxida a pardo claro, viscoso. Suele encontrarse en la parte baja del departamento, entre 950-1570 m, cerca de cuerpos de agua y zonas sombrías. Su distribución abarca la zona baja del municipio de Circasia, La Tebaida y Quimbaya.

Espécimen representativo. La Tebaida: Vda El Alambrado, Hda Balsora, río La Vieja, 1040 m, 13 mar 1985, Arbeláez 823 (HUQ).

17. Croton xalapensis Kunth Nov. Gen. Sp. (quarto ed.) 2: 85. 1817.

Árbol 4-12 m de alto, monoico. Látex amarillento a translúcido. Se distribuye principalmente en las zonas altas del departamento, en climas templados; hasta el momento se ha encontrado en los municipios de Armenia, Calarcá, Circasia, Filandia, Génova y Salento. C. xalapensis se localiza en altitudes mayores a los 1500 m, sin sobrepasar los 2950 $\mathrm{m}$., en bosques secundarios o fragmentos boscosos.

Espécimen representativo. Filandia: Vda Bambuco Alto, Fca La Esperanza, 1780 m, 30 jul 1987, Arbeláez et al. 2204 (HUQ).

\section{Croton sp.}

Árboles de 5-15(-25) m de alto, ca. $100 \mathrm{~cm}$ de CAP, monoico. Látex rojo, oxida a pardo oscuro. Croton sp. se ha encontrado en los municipios de Armenia, Calarcá, Circasia, Córdoba, Filandia, Pijao y Salento, siendo frecuentemente hallada en lugares sombríos, ya sea en bordes de bosques o en el interior de ellos. Su distribución se relaciona con la zona templada del departamento, comprendiendo desde los 1500-2200 m. Comentarios traxonómicos sobre esta especie pueden verse en la discusión.

Espécimen representativo. Filandia: Reserva Forestal Bremen, camino a Buenos Aires, 1800-2200 m, 25 may 1996, Gómez \& Murillo 30 (HUQ).

Dalechampia Plum. ex L. Sp. PI. 2: 1054. 1753. 1753.

Especie tipo. Dalechampia scandens L. Sp. PI. 2: 1054.

Etimología. Dalechampia es la latinización de la expresión francesa D'Aléchamps, nombre utilizado por el naturalista Charles Plumier en honor al médico, botánico, filósofo y naturalista francés Jacques Daléchamps (MacBride 1951).

Webster (1994) reporta aproximadamente 100 especies para el mundo, distribuidas en el trópico, 90 de las cuales son americanas, 20 son africanas y una es asiática. Para Colombia, Murillo (2004) reporta un estimativo de 11 especies. Para el Quindío, no se habían realizados reportes en la anterioridad, encontrándose en esta investigación tres nuevo registros para la zona, $D$. canescens, $D$. cissifolia y $D$. dioscoreifolia.

19. Dalechampia canescens Kunth Nov. Gen. Sp. (quarto ed.) 2: 98. 1817.

Liana escandente, monoica, latex ausente. $D$. canescens ha sido encontrada en los municipios cálidos y bajos del departamento tales como La Tebaida, Quimbaya y Montenegro. Según la ubicación de todos los ejemplares, se puede observar que se encnuetra relacionada de forma específica con la cuenca del río La Vieja y los ecosistemas aledaños.

Espécimen representativo. Montenegro: Ribera del río La Vieja, puente La María, 1000 m, 6 may 1984, Arbeláez et al. 338 (HUQ).

20. Dalechampia cissifolia Poepp. Nov. Gen. Sp. PI. 3: 20. 1845.

Liana, monoica, látex ausente. Esta especie solo se ha encontrado en el municipio de Quimbaya, a una altitud de $1340 \mathrm{~m}$, lindando con un guadual.

Espécimen representativo. Quimbaya: Vda La soledad, Fca El Perú, borde de guadual, 1340 m, 26 sep 1996, Vélez et al. 6319 (HUQ).

21. Dalechampia dioscoreifolia Poepp. Nov. Gen. Sp. PI. 3: 20. 1841. Figura 2.

Liana, monoica, látex ausente. La distribución de esta especie es limitada, en el departamento del Quindío que solo ha sido encontrada en los municipios de Circasia y Quimbaya. Al igual que $D$. canescens la distribución de esta especie parece estar asociada con la cuenca del rio la Vieja.

Espécimen representativo. Quimbaya: Vda La Española, Fca El Ocaso, bosque, 935 m, 31 jul 1997, Agudelo et al. 3565 (HUQ).

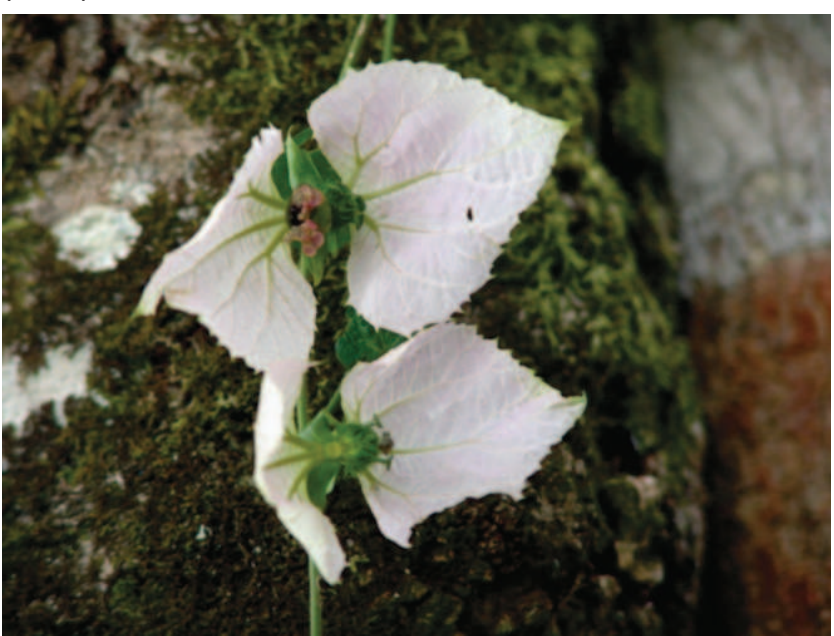

Figura 2. Detalle inflorescencia de Dalechampia dioscoreifolia

Rev. Invest. Univ. Quindío (20): 126 - 136. Armenia - Colombia 
Dysopsis Baillon, Etude Gén. Euphorb. 435. 1858.

Especie tipo. Dysopsis glechomoides (A. Rich.) Müll. Arg. Prodr. 15(2): 949. 1866.

Etimologia. Dysopsis proviene de la unión de dos palabras griegas, dys que significa ausencia, impedimento o dificultad; y opsis que hace referencia a ver, visualizar o generar imágenes mentales; significando en conjunto, difícil de observar.

Dysopsis está constituido por tres especies distribuidas en Suramérica y Centroamérica (Lozano \& Murillo 2001). Para Colombia, Murillo (2004) reporta una especie, siendo la misma que se encontró para el Quindío en este trabajo, Dysopsis paucidentata.

22. Dysopsis paucidentata (Müll. Arg.) Lozano y J. Murillo en Lozano, G. \& Murillo, J. Caldasia 23(2):419-426. 2001. Fig. 52 y 53.

Hierba monoica postrada. Látex ausente. Solo se ha encontrado en el municipio de Salento, entre los $3180 \mathrm{~m}$ y 3600 m, en zonas muy húmedas, creciendo en compañía de plantas del género Hydrocotyle (Apiaceae).

Espécimen representativo. Salento: Camino de la estación biológica Estrella de Agua al páramo de Frontino, 3180-3600 m, 21 jun 1994, Vélez et al. 3994 (HUQ).

Euphorbia L. Sp. PI. 1: 450-463. 1753; Candolle, P. Prodromus Systematis Naturalis Regni Vegetabilis 15(2): 106. 1862.

Especie tipo. Euphorbia antiquorum L. - Species Plantarum 1:450.1753.

Etimología. La palabra Euphorbia proviene de Euphorbus, nombre del médico oficial del rey Juba II de Mauritania (53 a.C.), quien frecuentemente usaba el látex de Euphorbia resinifera (anteriormente llamada E. regisjubae) para tratar enfermedades y heridas (Pérez 1965).

Es un género principalmente tropical o subtropical, compuesto por ca. 1800 especies (Webster et al. 1999). En la revisión que hace Murillo (2004) al herbario COL, reporta 43 especies de este género para Colombia; él en este listado vuelve a considerar el tratamiento de Chamaesyce como subgénero de Euphorbia, realizando un ejercicio de identificación de sinonimias, razón por la cual se puede decir que en realidad Murillo reportó ca. 38 especies de Euphorbia s.s. En este trabajo, se repotan 9 especies $E$. cotinifolia, E. graminea, E. goudotii, E. heterophylla, E. hoffmanniana, E. leucocephala, E. milii, E. peplus y E. pulcherrima.

23. Euphorbia cotinifolia L. Sp. PI. 1: 453. 1753.

Arbusto 2.5-8 m de alto, monoico. Látex blanco, viscoso, oxida a rojizo pardo. E. cotinifolia por ser una planta cultivada, se encuentra cerca de fincas y áreas urbanas, en los municipios de Armenia, Buenavista, Calarcá, Córdoba y Quimbaya, ubicándose desde 1150-1750 m. Ademas ejemplares de esta especie, han sido observados en los municipios de Salento, Génova, Circasia, Montenegro, La Tebaida.

Espécimen representativo. Buenavista: Vda La Granja, Fca Mi Casa, borde de la carretera, 1750 m, 18 nov 1997, Ochoa \& Modera67(HUQ).

24. Euphorbia graminea Jacq. Select. Stirp. Amer. Hist. 151.
1763.

Hierba $15-60 \mathrm{~cm}$ de alto, monoica, erecta a levemente postrada, ramificada dicotómica a simpódicamente. Puede hallarse creciendo tanto en zonas con climas cálidos como templados, hasta el momento se ha localizado en Armenia, Córdoba, La Tebaida, Montenegro y Quimbaya, siempre asociadas a cultivos de Coffea arabiga. El rango altitudinal en donde se encuentra comprende $1150-1650 \mathrm{~m}$.

Espécimen representativo. Armenia: Vda La Patria, borde de camino, 1500 m, 21 sep 1995, Vélez et al. 5761 (HUQ).

25. Euphorbia goudotii Boiss. Prodr. 15(2): 106. 1862. Hierba escandente, 60-1.5 m de alto, ramificada simpódicamente. Látex blanco, oxida a verde pardo. Se encuentra en bordes de caminos, teniendo preferencia por lugares cercanos a cuerpos de agua; se ha localizado en los municipios de Circasia y Salento, en una altitud promedio de $1530 \mathrm{~m}$.

Espécimen representativo. Circasia: Vda Barcelona Baja,

Fca Villa Mompasanos, bosque relictual, 1500 m, 13 dic 1994, Vélez et al. 4733 (HUQ).

26. Euphorbia heterophylla L. Sp. PI. 1: 453. 1753.

Hierba $10-65 \mathrm{~cm}$ de alto, ramificada apicalmente, pocas veces basalmente, monoica. Látex abundante, blanco, viscoso, oxida a pardo verdoso a rojizo. E. heterophylla se han localizado en todos los municipios del Quindío, mostrando así una alta capacidad para la adaptación a climas, suelos, condiciones freáticas y demas variaciones climáticas. El rango altitudinal en el cual se distribuye tambien es amplio, encontrándose desde 1000-1895.

Espécimen representativo. Buenavista: Vda La Huecada, Fca La Travista, malezas de cafetal, 1420 m, 23 jun 1991, Agudelo \& Correa 2309 (HUQ).

27. Euphorbia hoffmanniana (Klotzsch \& Garcke) Boiss. Prodr. 15(2): 99. 1862.

Hierba escandente, 1-2 m de alto, ramificada monopodicamente. Látex blanco, oxida a verde pardo. Hasta el momento solo se le ha localizado en el municipio de Salento; aspectos puntuales sobre la distribución y hábitat de dicha especie se desconocen por solo estar representada por un espécimen.

Espécimen representativo. Salento: Reserva natural Alto Navarco, s.a., 22 mar 1992, Vélez \& Ocampo 2810 (HUQ).

28. Euphorbia leucocephala Lotsy Bot. Gaz. 20(8): 350, t. 24. 1895.

Hierba ca. $50 \mathrm{~cm}$ de alto, monoica. Látex blanco. Se ha encontrado solamente en el municipio de Quimbaya en una altitud aproximada a $1310 \mathrm{~m}$.

Espécimen representativo. Quimbaya: Vda El Laurel, Fca La Primavera, malezas de cafetal, 1310 m, 16 sep 1991, Agudelo \& Abril 2526 (HUQ).

29. Euphorbia milii Des Moul. Bull. Hist. Nat. Soc. Linn. Bordeaux 1(1): 27-30, pl.1.1826.

Hierba postrada, gregaria, monoica, 20-50(-70) cm de alto; crecimiento horizontal predominante. Látex blanco, oxida a verde pardo. Su distribución actual se centra en locaciones urbanas de Armenia, La Tebaida, Montenegro, Calarcá, Buenavista, Córdoba y Circasia, siendo su distribución supeditada por el interés antropológico, debido a su único 
uso ornamental.

Espécimen representativo. Armenia: Calle 18 con carrera 17, 1525 m, 7 jun 1996, Duque J 2 (HUQ).

30. Euphorbia peplus L. Sp. Pl. 1: 456. 1753.

Hierba 20-30 cm, monoica, ramificada dicotómicamente. Se ha encontrado en los municipios de Filandia y Salento, estando asociada a las temperaturas templadas, hallándose en altitudes entre 1900-1970 m.

Espécimen representativo. Salento: Cementerio de Salento, 1970 m, 19 dic 1984, Arbeláez et al. 551 (HUQ).

31. Euphorbia pulcherrima Willd. ex Klotzsch Allg. Gartenzeitung 2(4):27-28. 1834.

Arbusto ampliamente ramificado, monoico, 0.6-3(-6.5) cm de alto, algunas veces escandente. Látex viscoso, blanco a blanco rojizo, oxida a pardo claro. La distribución de esta especie se encuentra limitada a centros urbanos.

Espécimen representativo. Armenia: Límites con Circasia, 1600 m, 8 jul 1979, Carvajal et al. 71 (HUQ).

Jatropha L. Sp. PI. 2: 1006-1007. 1753.

Especie tipo. Jatropha gossypiifolia L. Sp. PI. 2: 1006. 1753.

Etimología. Jatropha de las palabras griegas iatrós, doctor y trophé, comida, refiriéndose a las cualidades nutricionales de algunas de estas plantas cuando son consumidas en cantidades pequeñas. (Contr. Gard. Dave, 2007)

Jatropha es un género de distribución pantropical, subtropical y norteamericano, con aproximadamente 175 especies (Webster et al. 1999). En Colombia Murillo (2004), estima un total de 6 especies. Para el Quindío, en este trabajo se reportan dos especies, Jatropha integerrima y J. multifida siendo ellas de uso ornamental.

32. Jatropha integerrima Jacq. Select. Stirp. Amer. Hist. 256, pl. 183, f. 47. 1763.

Arbustos monoicos, de hasta $2 \mathrm{~m}$. Látex blanco. Las plantas de J. integerrima, no suelen ser muy comunes en el departamento, solo se encuentran en jardines y lugares adecuados con plantas Ilamativas. Hasta el momento solo se ha localizado en el municipio de Armenia, a una altitud de $1540 \mathrm{~m}$.

Espécimen representativo. Armenia: Universidad del Quindío, 1540 m, 30 may 2000, Santa 5 (HUQ).

33. Jatropha multifida L. Sp. PI. 2: 1006-1007. 1753.

Arbusto de hasta $3 \mathrm{~m}$ de alto, ramificado basalmente, monoico. Látex blanco, oxida a blanquecino verdoso, ligeramente viscoso. Esta especie por ser ornamental, está destinada a ser encontrada en regiones pobladas, ya sean urbes o zonas rurales.

Espécimen representativo. Armenia: Universidad del Quindío, 1540 m, 20 nov 1995, Gallego 1 (HUQ).

Mabea Aubl. Hist. Pl. Guiane 2: 867. 1775.

Especie tipo. Mabea piriri Aubl. - Histoire des plantes de la Guiane Françoise 2: 867, t. 334, f. 1. 1775.

Etimología. Mabea deriva del nombre vernacular usado por los indígenas de la Guyana Francesa Mabé, para referirse a plantas de este género, Aublet por su parte latinizó este vocablo cuando describió este taxón en 1775. (comm. pers. Esser)

Es un género diverso, constituido por ca. 50 especies neotropicales (Webster 1994). Para Colombia se tienen reportadas 18 especies, distribuidas principalmente en los andes colombianos (Murillo 2004). En este trabajo se reportan dos especies, Mabea klugii y M. montana.

34. Mabea klugii Steyerm. Field Mus. Nat. Hist., Bot. Ser. 17(5): 416. 1938.

Árbol, 6-15 $\mathrm{m}$ de alto. Látex blanco. M. klugii puede encontrarse en el Quindío en municipios tales como Buenavista y Pijao, en zonas principalmente altas, entre los 1800 y los $2040 \mathrm{~m}$.

Espécimen representativo. Buenavista: Carretera que conduce a Cumaral, Vda Varsovia, Hda Varsovia, 1860 m, 17 mar 1988, Arbeláez et al. 2481 (HUQ).

35. Mabea montana Müll. Arg. Prodr. 15(2): 1151. 1866. Árbol ca. $5 \mathrm{~m}$ de alto. Látex blanco abundante. Esta especie solamente ha sido localizada en el municipio de Buenavista al sur del Quindío, a una altura de $1790 \mathrm{~m}$. No se descarta la posibilidad de encontrarlo en las zonas de la misma altitud en Córdoba y Pijao.

Espécimen representativo. Buenavista: Vda El Balso, Fca La Lecheria, 1790 m, 27 sep 2004, Viveros et al. 740 (HUQ).

Manihot Mill. Gard. Dict. Abr. (ed. 4) 28. 1754.

Especie tipo. Manihot esculenta Crantz Inst. Rei Herb. 1: 167. 1766.

Etimología. El nombre de este género (Manihot) proviene de la latinización de la palabra brasilera Manioc, el cual se aplica a algunas plantas de este género (Contr. Gard. Dave, 2007). Es un género constituido por ca. 100 especies distribuido principalmente en América (Rogers \& Appan 1973). Para Colombia se han reportado 4 especies (Murillo 2004). En el Quindío solo se encontró una especie, Manihot esculenta la cual se encuentra como cultivada.

36. Manihot esculenta Crantz Inst. Rei Herb. 1: 167. 1766. Arbusto monoico hasta de $2 \mathrm{~m}$ de alto, ramificado basalmente, ramas postradas, penduladas. Látex blanco, oxida a pardo rojizo. $M$. esculenta es ampliamente en el departamento sobretodo en las zonas cálidas, incluyendo a los municipios de Montenegro, Quimbaya y La Tebaida, además algunas zonas bajas de Calarcá y Armenia. Generalmente estos cultivos se encuentran asociados a cafetales y a zonas en recuperación. El intervalo de altitudes en el cual se puede ubicar la especie es 950-1500 m.

Espécimen representativo. Quimbaya: Vda Puerto Alejandría, camino, borde de carretera, 980 m, 13 nov 1996, Angel \& Torres 17(HUQ).

Plukenetia L. Sp. PI. 2: 1192. 1753.

Especie tipo. Plukenetia volubilis L. -Sp. PI. 2: 1192. 1753.

Etimología. Este género fue nombrado en honor a Leonard Plukenet, botánico e ilustrador britanico de la revista Phytographia (Contr. Gard. Dave 2007).

El género completo consta de aproximadamente 17 especies, 12 de ellas localizadas en el Neotropico, cuatro en America y Madagascar, una en Asia (Gillespie \& Armbruster 1997). Murillo (2004) registra para Colombia 4 especies de Plukenetia. En este trabajo se reporta una especie $P$. volubilis siendo reporte nuevo para el departamento.

37. Plukenetia volubilis L. Sp. PI. 2: 1192. 1753. 
Liana escandente, voluble a rígida. Látex ausente. P. volubilis se ha encontrado unicamente en las partes altas del departamento, por encima de los $1500 \mathrm{~m}$, en los municipios de Armenia, Filandia, Circasia y Salento. Suelen encontrarse en borde de bosques secundarios, en lugares sombríos y húmedos. Es una especie que suele ser cultivada con fines medicinales.

Espécimen representativo. Armenia: El Pórtico, hacia adentro, 1500 m, 9 ene 1997, Vargas 3411 (HUQ).

Ricinus L. Sp. Pl. 2: 1007. 1753.

Especie tipo. Ricinus communis L. - Species Plantarum 2: 1007.1753.

Etimología. La expresión Ricinus proviene del nombre en latín de una garrapata que parasita ovejas del Mediterráneo, y fue dado a estas plantas por la semejanza que tienen sus semillas con dichos animales. (Contr. Gard. Dave 2007).

Género nativo de África del este o de la India, cultivado y naturalizado a lo largo de todo el trópico y los subtrópicos, solo cuenta con una especie Ricinus communis (Webster et al. 1999). Murillo (2004) reporta dicha especie para algunas zonas de Colombia, y en este trabajo se incluye la misma para el departamento.

38. Ricinus communis L. Sp. PI. 2: 1007. 1753.

Arbustos monoicos, 3-5 m de altura, 9-12 cm de CAP. Látex ausente, capa cerosa blanquecina, en hojas y pecíolos. De Ricinus communis se estudiaron ejemplares encontrados en los municipios de Armenia, Montenegro y Quimbaya, tierras que varían de clima entre cálido y templado, comprendiendo altitudes entre $950-2500$ m; no obstante, se han observado individuos en todos los demás municipios, siendo poco ocurrentes en Salento y en Córdoba. Debido a que es una especie altamente cultivada su distribución no responde a un patrón natural.

Espécimen representativo. Montenegro: Vda Veraguas, parte baja del bosque de Veraguas, 950 m, 10 may 2006, Maya 14 (HUQ).

Sapium Jacq. Enum. Syst. PI. 9, 31.1760.

Especie tipo. Sapium jamaicense Sw. - Adnotationes Botanicae 62.1829.

Etimología. Sapium corresponde en latín antiguo al nombre de un pino resinoso, en el cual, la espuma de su savia semejaba a la espuma del jabón (Contr. Gard. Dave 2007). Es un género principalmente pantropical con 90-100 especies, en su mayoría neotropicales (Webster 1994); aunque Krujit (1989), consideró, al género como exclusivamente neotropical compuesto por 21 especies. Murillo (2004) realizó un listado en donde incluye 6 especies. Dentro de este trabajo, se reporta a Sapium stylare como la única especie del género encontrada hasta el momento en el departamento del Quindío.

39. Sapium stylare Müll. Arg. Linnaea 32: 119. 1863.

Árbol 4-15(-20) m de alto, ca. $25 \mathrm{~cm}$ de circunferencia. Látex blanco, oxida a pardo o verdoso. Sapium stylare se encuentra restringido a zonas altas del departamento, incluyendo los municipios de Armenia, Calarcá, Circasia, Filandia, Génova, Quimbaya y Salento, comprendiendo altitudes no menores a los $1500 \mathrm{~m}$, hallándose en pisos térmicos altos a los $3000 \mathrm{~m}$, siendo así la Euphorbiaceae quindiana de mayores altitudes, después de Dysopsis paucidentata que se ubica en esta franja altitudinal. Espécimen representativo. Génova: Camino Fca Las MirlasServia, 2400-2700 m, 29 abr 1990, Vélez et al. 1328 (HUQ).

Tetrorchidium Poeppig en Poepp. \& Endl., Nov. Gen \& Sp. 3:23, t. 227. 1845

Especie tipo. Tetrorchidium rubrivenium Poepp. Nov. Gen. Sp. Pl. 3: 23. 1841.

Etimología. El nombre se refiere a los estambres con cuatro tecas, del prefijo griego tetra, cuatro y de la palabra orchis, testículo, característicos de este género (Cuatrecasas 1957). Comprende ca. 20 especies, 15 de ellas se encuentran distribuidas en América tropical (sect. Tetrorchidium) y 5 en Africa (sect. Hasskarlia). Cuatrecasas (1957) y luego Murillo (2004) reportan para Colombia 7 especies, distribuidas principalmente en la región Andina. En este trabajo se encontró una especie, Tetrorchidium rubrivenium reduciendo el número de especies esperado por Vargas (2002), de dos especies, puesto que los ejemplares determinados como T. boyacanum no coresponden a dicho nombre, por carecer de indumento tomentoso y por tener menos de 6 estambres.

40. Tetrorchidium rubrivenium Poepp. en Poepp. \& Endl., Nov. Gen \& Sp. 23:227, 1845, 31.

Árboles, arbustos, dioicos, 5 a 12 m, con un CAP de 13 a 15 $\mathrm{cm}$. Látex transparente. Se encuentra en los municipios de Armenia, Calarcá, Montenegro, Pijao y Quimbaya, en zonas correspondientes a clima cálido con poca pluviosidad, entre los 1000-1300 m, siendo excepcional hallarlo a los $1650 \mathrm{~m}$.

Espécimen representativo. Pijao: Margen izquierda de carretera a Caicedonia $2 \mathrm{~km}$ antes de Barragán, Fca Las Acacias, 1140 m, 21 ago 1985, Arbeláez 967 (HUQ).

\section{DISCUSIÓN}

Con respecto a Croton sp, los ejemplares estudiados se encontraban bajo el nombre de Croton magdalenensis, sin embargo se considera necesario tomar con cautela este nombre ya que al momento de determinarlos, surgieron algunos problemas bibliográficos; Croton magdalenensis, es una de las especies más conocidas en la región del eje cafetero, sin embargo no se encuentran libros de importancia taxonómica que la citaran, excepto el catálogo de plantas de Ecuador (Smith et al. 1999), careciendo este de una clave y una descripción adecuada que pudieran servir de apoyo para la confirmación de este nombre. Haciendo retrospección, se logró encontrar el protólogo (Müll. Arg. 1864), en donde detalla una especie de Croton, siendo amplio en su descripción, dando cabida a algunas especies como $C$. xalapensis y $C$. draco, razón por cual se considera que este protólogo no aporta información contundente para poder determinar los ejemplares bajo este nombre, por lo cual se tomó la decisión de dejarlos determinados como Croton sp, es decir como taxón en espera para la definición de su epíteto y poniendo de manifiesto la irregularidad del nombre mismo, el cual pueda ser una sinonimia de otra especie.

En algunas revisiones taxonómicas, se encuentra la descripción de Acalypha amentacea como A. wilkesiana siendo este una sinonimia considerada anteriormente como valida (Fosberg \& Sachet 1980), sin embargo en una revisión reciente, Webster et al. (2001) la tratan de 
nuevo como A. wilkesiana, sin dar a conocer ningún argumento; por lo tanto, en este trabajo se mantiene el nombre propuesto por Fosberg \& Sachet (1980).

Reconocer Alchornea glandulosa puede ser confuso, ya que usando caracteres foliares, semeja a otras especies del género ( $A$. grandiflora, $A$. grandis, $A$. glandulosa y $A$. coelophylla), sin embargo, el número de glándulas (6-10) en la base de la hoja, permiten descartar grupos cercanos; pero diagnósticamente, las flores femeninas, son las que permiten diferenciarla de otras, el ovario tomentoso, estilos fuertemente muricados, $1-2 \mathrm{~cm}$ de largo, son en sí las características definitivas para su diferenciación. A. latifolia, se considera para esta revisión que el estilo solo abarca la porción recta inserta en el ovario directamente, debido a que a partir de él se despliegan dos estructuras con textura, color y forma diferente, la cual se podría considerar como área estigmática, sabiendo que en la literatura estudiada se nombran a los estigmas como si fueran estilos, se hace una reflexión a cerca de la necesidad de estudiar dicha estructura, para esclarecer su nomenclatura.

Burger \& Huft (1995) consideran que las flores femeninas de A. glandulosa son sésiles, pero en realidad pueden ser subpedíceladas, se puede ver claramente cuando pasa de estado maduro a fruto, el cual está sostenido por un pequeño pedúnculo, menor a $2 \mathrm{~mm}$, por lo tanto el tratamiento de inflorescencias espiciformes en su manuscrito es incorrecto. El mismo tratamiento lo hacen Webster \& Burch (1968), retomándolo luego en Webster \& Huft (1988); en Flora de Panamá y su posterior revisión, en los cual se nombran a las inflorescencias femeninas como espigas, sin tener en cuenta su propia consideración de que las flores femeninas son subsésiles.

Dalechampia cissifolia fue tratada por Standley \& Steyermark (1949) en Flora de Guatemala de dos formas, dividiéndose los ejemplares en dos grupos diferentes a los cuales se les adjudicaron nombres independientes, $D$. panamensis y D. heteromorpha. MacBride (1951) citó y describió algunos ejemplares bajo el nombre de $D$. cissifolia, haciendo énfasis en su sinonimia anterior ( $D$. triphylla). Luego Webster (1967) en una revisión hecha al género, ubicó a $D$. panamensis como subespecie de $D$. cissifolia. Basados en el trabajo anterior Webster \& Burch (1968) y Webster \& Huft (1988) trataron morfotipos similares como D. cissifolia ssp. panamensis en la Flora de Panamá. Para Flora Costaricensis, Burger \& Huft (1995), citan a D. cissifolia como nombre aceptado de las anteriores sinonimias, pero continúan considerando a $D$. heteromorpha como especie diferente de $D$. cissifolia, al igual que lo considerado por Standley \& Steyermark en 1949. D. heteromorpha por otro lado, cambió su ubicación y actualmente es considerada sinonimia, al igual que $D$. panamensis de $D$. cissifolia, este cambio es expuesto por Webster et al. (2001) en Flora de Nicaragua. Sin duda alguna, el complejo Triphylla es un grupo que debe ser estudiado con detenimiento y considerar la posibilidad de sinonimización de algunos taxones tal y como Allem \& Waecher (1977) sugieren.

Vargas (2002), reporta a P. polydenia para el Quindío, siendo errado este reporte ya que en su manuscrito la describe como una planta trepadora con hojas cordiformes, lo que implica, hojas con lámina ovada y base bilobulada o cordada; ambas características correspondientes a $P$. volubilis; de este modo, contrastando con los verdaderos rasgos de $P$. polydenia, la cual tiene hojas elípticas con base de redondeada a obtusa.

\section{CONCLUSIONES}

En esta lista anotada se reporta la presencia de 40 especies, en 16 géneros; representando así el $10.26 \%$ de las especies estimadas para Colombia y el $20.51 \%$ de los géneros colombianos, tales porcentajes pueden considerarse altos, ya que el Quindío representa solo el $0.2 \%$ del área continental Colombiana.

De los géneros encontrados, el que presentó un mayor número de especies fue Euphorbia (9 spp); seguido por Acalypha (7 spp), Croton (5 spp) y Dalechampia (3 spp). Chamaesyce, Jatropha y Alchornea fueron géneros pequeños, representados cada uno de ellos por 2 especies; $y$ por último se hallaron 8 géneros monoespecíficos: Manihot, Plukenetia, Ricinus, Sapium, Tetrorchidium, Dysopsis, Codiaeum y Caperonia.

De las 40 especies registradas, 14 (35\%) son nuevos reportes para el Quindío, Acalypha setosa, A. amentacea, Codiaeum variegatum, Croton speciosus, C. xalapensis, Dalechampia canescens, D. cissifolia, Euphorbia graminea, E. leucocephala, Euphorbia peplus, Jatropha multifida, J. integerrima, Mabea montana y Plukenetia volubilis. Croton xalapensis es además un nuevo registro para Colombia.

Dentro de las especies encontradas en el Quindío, Acalypha diversifolia, A. macrostachya, y Euphorbia heterophylla, Euphorbia cotinifolia y Ricinus communis son las especies con más amplia distribución en el departamento, hallándose desde los 1000-2200 m, en todos los municipios. Mientras que las Dysopsis paucidentata, Caperonia palustris, Dalechampia cissifolia, D. canescens, D. dioscoreifolia, Euphorbia peplus, E. leucocephala, E. hoffmanniana, E. goudotii, Mabea montana, Acalypha platyphylla, $A$. setosa, presentarón distribución más restringida. 


\section{BIBLIOGRAFÍA}

1. Webster, G.L., P.E. Berry, W.S. Armbruster, H,J. Esser, L.J. Gillespie, W.J. Hayden, F.A. Levin, R.S. Secco, \& S.V. Heald. 1999. Euphorbiaceae. En: Berry, P.E., K. Yatskievych \& B.K. Holst (eds.). Fl. of the Venezuelan Guayana 4:74-228.

2. Maya L., C.A. \& C.A. Agudelo. 2007. Inventario taxonómico de la familia Euphorbiaceae para el departamento del Quindío. Universidad del Quindío. Trabajo de grado.

3. Villarreal H., M. Álvarez, S. Córdoba, F.Escobar, G. Fagua, F. Gast, H. Mendoza, M. Ospina \& A.M. Umaña. 2006. Manual de métodos para el desarrollo de inventarios de biodiversidad. Segunda edición. Programa de Inventarios de Biodiversidad. Instituto de Investigación de Recursos Biológicos Alexander von Humboldt. Bogotá, Colombia. 236 pp.

4. Lot, A. \& F. Chiang. (Comp). 1986. Manual de herbario. Administración y manejo de colecciones, técnicas de recolección y preparación de ejemplares botánicos. led, México, D.F. Ed. Consejo Nacional de la flora de México. A.C. 142 pp.

5. Rentería I. 1994 Contribución al conocimiento del género Alchornea Sw. (Euphorbiaceae) en Colombia. Trabajo de grado, Universidad Nacional de Colombia, Bogotá.

6. Cuatrecasas, J. 1957. The Colombian species of Tetrorchidium. Brittonia 9:76-82.

Croat, T.B. 1978. Flora of Barro Colorado Island. Stanford University Press, Stanford, California. 943 pp.

7. Standley, P.C. \& J.A. Steyermark. 1949. Flora of Guatemala. Fieldiana: Botany 24. Part VI. 1-438.

8. Webster, G.L. \& D. Burch, 1968. Euphorbiaceae. Págs. 211-350 en: Woodson, R. E., Schery, R. W. \& Collaborators.1968. Flora of Panamá. Ann. Missouri Bot. Gard. 54.

9. Cardiel, J.M. 1995. Flora de Colombia, 15. Acalypha (Euphorbiaceae). Impreso en Colombia por Juan Pablo Arbeláez. Santa Fé de Bogotá. 153 pp.

10. Macbride, J.F. 1951. Euphorbiaceae. In: MacBride, J. F. Flora of Peru. Publ. Field Mus. Nat. Hist., Bot. Ser. 8 part. 3A(1):3200.

11. Webster, G.L. 1994. Synopsis of the genera and suprageneric taxa of Euphorbiaceae. Ann. Missouri Bot. Gard. 81(1):33144.

12. Pax, F., K Hoffmann. 1914. Euphorbiaceae-Acalypheae-Mercurialinae mit 317 Einzelbildern in 67 Figuren unter Mitwirkung. In A. Engler, editor, Das Pflanzenreich IV. 147 VII (Heft 63).

13. Secco, R.S. 1999. Uma espécie e uma combinação novas de Alchornea Sw. (Euphorbiaceae) da Bolivia. Revta brasil. Bot, 22(2):141-146.

14. Murillo, J.C. 2004. Las Euphorbiaceae de Colombia. Biota Colombiana 5(2):183-200.

15. Vargas W.G. 2002. Guía ilustrada de las plantas de las montañas del Quindío y los Andes Centrales. Manizales. Universidad de Caldas. Centro Editorial. $813 \mathrm{pp}$.

16. Smith, L.B., R. Downs \& R.M. Klein. 1988. Euforbiáceas. In: Reitz, R. (ed.), Flora llustrada Catarinense EUFO: $174-192$.

17. Contribuidores de Dave's Garden. 2007. Botanary. Online Botany dictionary, extended service of Dave's Garden. [consultado mayo 2007]. Sitio: http://davesgarden.com/botanary/.

18. Lozano, G. \& J.C. Murillo. 2001. El género Dysopsis (Euphorbiaceae). Caldasia 23(2):419-426.

19. Pérez, E. 1965 (fecha de la primera edición). Plantas Utiles de Colombia, 14a edición. Editorial Víctor Hugo. Bogotá. Colombia. 1990.

20. Rogers, D.J. \& S.G. Appan. 1973. Manihot, Manihotoides (Euphorbiaceae). Flora Neotropica 13: 1-272.

21. Gillespie, L. \& W.S. Armbruster. 1997. A Contribution to the Guianan Flora: Dalechampia, Haemutostemon, Omphalea, Pera, Plukenetia and Tragia (Euphorbiaceae) with Notes on Subfamily Acalyphoideae. Smithsonian Contr. Bot. 86:1-48.

22. Krujit, R.C. 1989. Monographic studies on Sapium (Euphorbiaceae, Hippomaneae) and related genera. Sin publicar. Disertación doctoral (Ph.D. dissertation). Rijksuniversiteit, Utrecht.

23. Smith, L.B., G.L. Webster \& G. Mcpherson. 1999. Euphorbiaceae. En: Jørgensen, P. M. \& S. León-Yánez (eds.). 1999. Catalogue of the vascular plants of Ecuador. Monogr. Syst. Bot. Missouri Bot. Gard. 75:1-1182.

24. Müeller Argoviensis, J. 1864. Neue Euphorbiaceen des Herbarium Hooker in Kew. In: Bayerische botanische Gesellschaft, Regensburg. Flora oder Allgemeine botanische Zeitung. Vol. 47.642 pp.

25. Fosberg, F.R \& H. Sachet. 1980. Systematic studies of Micronesian plants. Smithsonian Contr. Bot. 45:1-40.

26. Webster, G.L., M.J. Huft \& G.A. Levin. 2001. Euphorbiaceae. In: Stevens, W. D., C. Ulloa U., A. Pool \& O. M. Montiel. (eds.). Monographs in Systematic Botany From the Missouri Botanical Garden. (Acceso web online: http://mobot.mobot.org/W3T/Search/Nicaragua/projsflnic.html)

27. Burger, W. \& M. Huft. 1995. Euphorbiaceae. In: Burger, W. (ed.). Flora Costaricensis. Fieldiana, Botany New Series 36:1169.

28. Webster, G.L. \& M.J. Huft, 1988. Revised Synopsis of Panamanian Euphorbiaceae. Ann. Missouri Bot. Gard. 75(3):10871144.

29. Webster, G.L. 1967. Two Neotropical Taxa of Dalechampia. Ann. Missouri Bot. Gard. 54:193-194.

30. Allem, A. C. \& Waecher, J. L. 1977. Notas sistemáticas y nuevos sinónimos en Euphorbiaceae de América del Sur - II. Rv. Brasil. Biol., 37(1): 91-101. 